\title{
Gigahertz quantum key distribution with InGaAs avalanche photodiodes
}

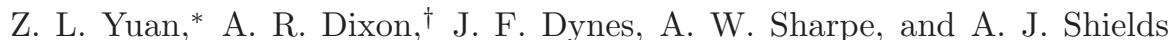 \\ Toshiba Research Europe Ltd, Cambridge Research Laboratory, \\ 208 Cambridge Science Park, Milton Road, Cambridge, CB4 0GZ, UK
}

(Dated: October 29, 2018)

\begin{abstract}
We report a demonstration of quantum key distribution (QKD) at GHz clock rates with InGaAs avalanche photodiodes (APDs) operating in a self-differencing mode. Such a mode of operation allows detection of extremely weak avalanches so that the detector afterpulse noise is sufficiently suppressed. The system is characterized by a secure bit rate of $2.37 \mathrm{Mbps}$ at $5.6 \mathrm{~km}$ and $27.9 \mathrm{kbps}$ at $65.5 \mathrm{~km}$ when the fiber dispersion is not compensated. After compensating the fiber dispersion, the QKD distance is extended to $101 \mathrm{~km}$, resulting in a secure key rate of $2.88 \mathrm{kbps}$. Our results suggest that InGaAs APDs are very well suited to GHz QKD applications.
\end{abstract}

Quantum key distribution (QKD) [1, 2, 3, 4] uniquely allows two remote parties (Alice and Bob) to exchange cryptographic keys with verifiable secrecy. Currently, much effort has been focused on increasing the key rate, [5, 6, 7, 8, 8, 9, 10, 11] towards the goal of key exchange being fast enough to offer information-theoretically secure encryption of high speed data communication. QKD with GHz clock rates 7, 8, 9] has already been demonstrated in various optical fiber based systems with elevated key rates. However, due to lack of practical detectors at telecom wavelengths, periodically poled $\mathrm{LiNbO}_{3}$ upconversion 8, 9] or superconducting nanowire detectors, [7] had to be used. Upconversion detectors suffer from high background count rates, while superconducting devices require cryogenic cooling to a few K. Neither of these devices are ideal for real-world applications.

Although widely used in the fiber-based $\mathrm{MHz}$ QKD systems, [11, 12, 13, 14] InGaAs avalanche photodiodes (APDs) have been generally regarded as unsuitable for $\mathrm{GHz}$ applications due to their severe afterpulsing 15. problem. Recently, a self-differencing technique has been shown to suppress the afterpulse noise at high gate frequencies. [16] It allows detection of weak avalanches

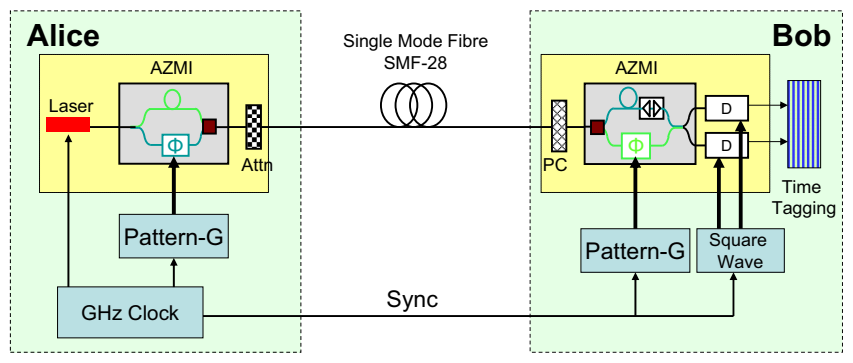

FIG. 1: QKD experimental setup. AZMI: asymmetric MachZender interferometer; Attn: optical intensity attenuator; PC: polarization controller; D: InGaAs APD.

*Electronic address: zhiliang.yuan@crl.toshiba.co.uk

${ }^{\dagger}$ Also at Cavendish Laboratory, University of Cambridge, J. J. Thomson Avenue, Cambridge CB3 OHE, UK. previously undetectable under conventional methods, and as a result, the afterpulse noise is sufficiently suppressed to allow APDs to be gated beyond $1 \mathrm{GHz}$. Moreoever, the self-differencing APD requires no cryogenic cooling, and features a high photon count rate $(100 \mathrm{MHz})$, low dead time $(<10 \mathrm{~ns})$ and low time jitter $(60 \mathrm{ps})$, suggesting it could be well suited to $\mathrm{GHz}$ QKD applications.

In this letter, we demonstrate, for the first time, $\mathrm{GHz}$ QKD with GHz-clocked InGaAs APDs. Secure key rates of $2.37 \mathrm{Mbps}, 684 \mathrm{kbps}$ and $27.9 \mathrm{kbps}$ have been achieved for fiber lengths of $5.6 \mathrm{~km}, 25.3 \mathrm{~km}$ and $65.5 \mathrm{~km}$ respectively. With fiber dispersion compensation, the QKD distance has been extended to $101 \mathrm{~km}$.

The key distribution system is based upon a time division Mach-Zender interferometer using phase modulation, 2, 12] as shown in Fig. 1. Photons are generated by a $1.55 \mu \mathrm{m}$ pulsed laser operating at $1.036 \mathrm{GHz}$. The optical pulses are strongly attenuated to the single photon level before leaving the sender's apparatus. Phase modulators in the two interfering arms are used for encoding. A polarization controller is used to recover photon polarizations before Bob's interferometer. Each detected photon is recorded with a unique time stamp using time-tagging electronics. Standard telecom fiber SMF-28 was used in this experiment, featuring a measured optical attenuation loss of $0.195 \mathrm{~dB} / \mathrm{km}$ at a wavelength of $1550 \mathrm{~nm}$.

Self-differencing circuits [16] were made with co-axial cables of precise lengths so as to match the laser clock frequency. Our InGaAs APD detectors are cooled typically to $-30{ }^{\circ} \mathrm{C}$ and gated with a square wave of $6.0 \mathrm{~V}$ amplitude synchronized to the laser frequency. They have a detection efficiency of $\sim 10 \%$, dark count probability of $2.9 \times 10^{-6}-3.3 \times 10^{-5}$ and an afterpulse probability of less than $6 \%$, depending on the bias applied.

Timing performance is of paramount importance for high speed QKD. Figure 2 shows a time-resolved histogram of photon arrivals recorded with a laser illumination of 0.008 photons/pulse. Each photon peak is very sharp, with a full width at half maximum of $60 \mathrm{ps}$, suggesting precise timing measurements. Importantly, adjacent peaks are well separated with a 700 ps gap within which no photons were registered at all, due to the na- 


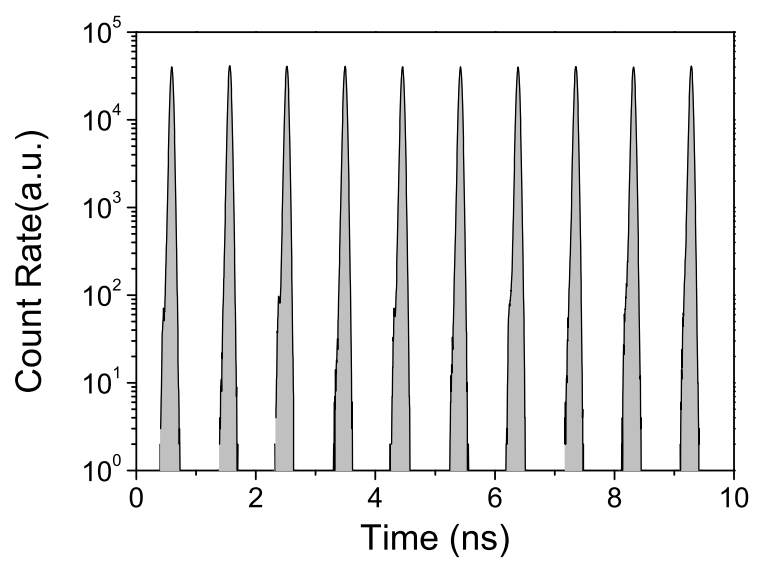

FIG. 2: Histogram of photon detections with illumination of an average pulse intensity of 0.008 photons at a repetition rate of $1.036 \mathrm{GHz}$.

ture of gated operation. Such a wide separation not only enables unambiguous bit assignment, but also aids considerable noise rejection.

For experimental simplicity, we choose to implement the standard BB84 protocol[1] with a fixed average optical pulse intensity of $\mu=0.2$. Time-tagged photon detection events are used to precisely measure the raw bit rate $\left(R_{\text {Raw }}\right)$ and the QBER $(e)$, from which the secure bit rate after privacy amplification can be determined using the formula 17] with a realistic error correction efficiency $f_{E C}=1.10,14$

$$
R_{\text {Secure }}=\frac{1}{2} R_{\text {Raw }}\left\{1-\left(1+f_{E C}\right) H(e)\right\}
$$

where $\frac{1}{2}$ represents the BB84 protocol efficiency, and $H(e)=-e \log _{2} e-(1-e) \log _{2}(1-e)$ is the binary Shannon entropy. 18]

Eqn. (1) would give an unconditionally secure 19 key rate if a truly single photon source were used. The multiphoton pulses, inevitably generated by the attenuated laser in weak pulse QKDs, open up a security threat in which an eavesdropper, Eve, can exploit these extra photons by performing a photon number splitting (PNS) attack. 20, 21] However, the PNS attack is currently unfeasible technologically, and more importantly it can be defeated by the recently developed decoy protocol. 22, 23. Our simulations suggest that the decoy protocol would allow an optimized pulse intensity of $\mu=0.52-0.69$, being considerably higher than our choice of $\mu=0.2$, thus ensuring that the QKD demonstrated below can be made unconditionally secure when required in future. From now on, the PNS attack is excluded from the security analysis.

To permit secure key distribution, the QBER must be lower than a certain threshold, which is $\sim 10 \%$ for our case. The sources contributing to the QBER can be ap-

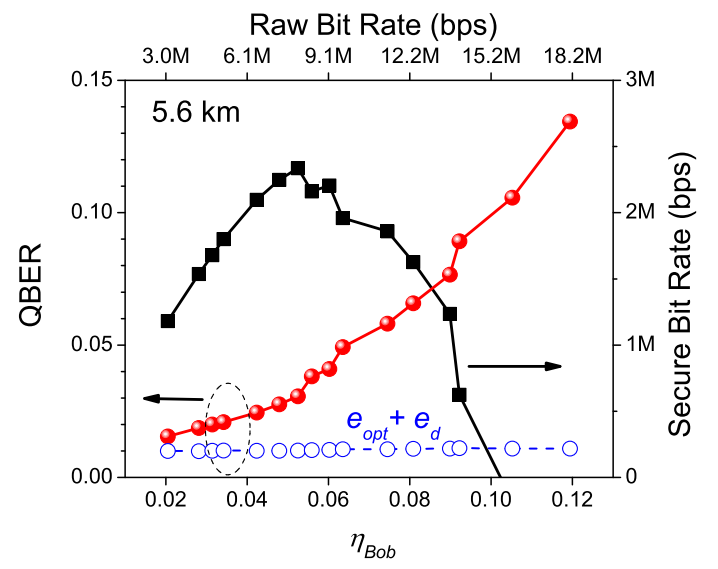

FIG. 3: The quantum bit error rate (QBER), and the secure bit rate as a function of Bob's detection efficiency $\eta_{B o b}$ (or raw bit rate) for a fiber link of $5.6 \mathrm{~km}$. Also shown is the QBER arising from the apparatus imperfection $\left(e_{o p t}\right)$ and detector dark counts $\left(e_{d}\right)$.

proximated as

$$
e \approx\left[e_{o p t}+\frac{1}{2} P_{a}\right]+\left[e_{d}+e_{b}\right]
$$

where $e_{o p t}$ is the error due to apparatus imperfections, such as interferometer mis-alignment and mismodulation; $P_{a}$ is the APD afterpulse probability, and the coefficient $\frac{1}{2}$ represents the fact that an afterpulse event has equal probabilities to produce a correct or an erroneous bit. These first two terms are fiber distance independent. $e_{d}$ is the contribution from the detector dark counts. $e_{b}$ is the interclock interference due to pulse broadening, existing only in high speed QKD systems, where an optical pulse may temporally spread into the neighboring clock cycle due to broadening caused by fiber dispersion. Both $e_{d}$ and $e_{b}$ are fiber length dependent and significant for long fibers, but are typically negligible at short fiber distances $(\leq 25 \mathrm{~km})$. $e_{\text {opt }}$ is estimated to be $0.9 \%$, based on the interferometer visibility $(99.4 \%)$ and mis-modulation. $P_{a}$ depends on the APD bias, 16] but was found to be below $6 \%$ when the detection efficiency is not greater than $10 \%$. As shown below, $P_{a}$ is the dominating error source.

It is clear from Eqn. (1) that a high raw bit rate and a low QBER are desirable to have a high secure bit rate. However, it is not possible to achieve both simultaneously. Increasing the raw bit rate by raising the detection efficiency always causes an increase in the QBER due to increased dark count rate and afterpulse probability. A trade-off must be made in order to achieve the optimal secure key rate. To find the optimal operating conditions, we tune the APD DC bias and hence vary the raw bit rate and the QBER. Figure 3 shows the measured QBER (solid circles) and the secure bit rate (solid square) for a 5.6-km fiber link as a function of Bob's detection efficiency $\eta_{B o b}$ (including Bob's optical loss). The raw bit 


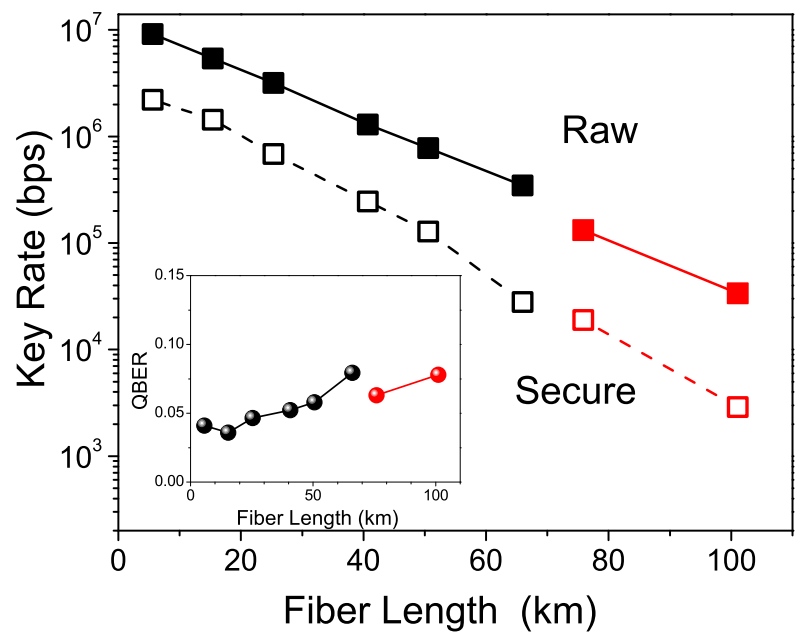

FIG. 4: Raw (solid squares) and secure (open squares) bit rates as a function of fiber length. The inset shows the quantum bit error rate (QBER). $75 \mathrm{~km}$ and $101 \mathrm{~km}$ results were obtained with pre-compensation of fiber chromatic dispersion.

rate increases six-fold from 3.1 Mbps to 18.2 Mpbs due to the increased detection efficiency. The QBER, which is as low as $1.55 \%$ at low detection efficiencies, increases monotonically, due to the increased noise, and eventually exceeds the security limit of $\sim 10 \%$. The QBER due to the optical imperfection $\left(e_{\text {opt }}\right)$ and the dark counts $\left(e_{d}\right)$ is also plotted, which makes only a small fraction of the total QBER at high detection efficiencies, suggesting that the detector afterpulse may be the dominating error source. The secure bit rate, calculated using Eqn. (1), is highest at an intermediate detection efficiency. The secure bit rate, which is $1.19 \mathrm{Mbps}$ at low detection efficiency, doubles to $2.37 \mathrm{Mbps}$ for a detection efficiency between $4 \%$ and $6 \%$. Further increasing the detector bias causes a sharp drop in secure bit rate, illustrating that the privacy amplification cost exceeds the gain in raw bit rate. The optimal detector bias was then used for fiber length dependence experiments, corresponding to $\eta_{B o b}=6.0 \%$, which gives the optimal secure bit rates over the fiber distances studied.

We perform QKD experiments over various fiber distances up to $65.5 \mathrm{~km}$. The raw bit rate, as shown in Fig. 4, decreases with fiber length at a rate of 0.24 $\mathrm{dB} / \mathrm{km}$. This rate is higher than the measured optical attenuation loss of $0.195 \mathrm{~dB} / \mathrm{km}$, because of the additional dispersion loss, as described later. The raw bit rate is $9.16 \mathrm{Mbps}$ at $5.6 \mathrm{~km}$ fiber, falling to $348 \mathrm{kbps}$ at $65.5 \mathrm{~km}$. The measured QBER is shown in the inset of Fig. 4. It remains fairly constant for short fibers below
$25 \mathrm{~km}$, but increases with fiber length for longer lengths due to a combination of the falling photon rate and the increasing deleterious dispersive pulse broadening. $e_{b}$ is estimated to be $2.1 \%$ for $65.5 \mathrm{~km}$ fiber, exceeding the contribution from the detector dark count $\left(e_{d}\right)$. Nonetheless, even without fiber dispersion compensation, the QBER at $65.5 \mathrm{~km}$ is well below the security limit $\sim 10 \%$, allowing secure keys to be formed. The calculated secure bit rate follows the raw bit rate, decreasing exponentially with fiber distance. The secure bit rate is determined to be $684 \mathrm{kbps}$, and $27.9 \mathrm{kbps}$ for 25.3 and $65.5 \mathrm{~km}$ respectively.

Secure key exchange over distances much longer than $65 \mathrm{~km}$ is not possible without compensation of dispersion of the fiber. The standard fibers used here have a chromatic dispersion of $17 \mathrm{ps} /(\mathrm{nm} \cdot \mathrm{km})$ at $1550 \mathrm{~nm}$. The chromatic dispersion of the fiber broadens the laser pulse, such that a fraction of photons are dispersed out of the APD detection gate. This causes the raw bit rate to drop more rapidly $(0.24 \mathrm{~dB} / \mathrm{km})$ with fiber length than expected from the optical attenuation $(0.195 \mathrm{~dB} / \mathrm{km})$. Furthermore, the fiber dispersion also causes temporal overlap of adjacent optical pulses, resulting in an increased interclock interference and error rate. Either effect deteriorates the QKD performance. In fact, the QBER is measured to be $17 \%$ for $75.8 \mathrm{~km}$ fiber, having exceeded the security limit, due largely to the contribution of the dispersive broadening $\left(e_{b}>10 \%\right)$.

We use a fiber Bragg grating device at Alice's side to pre-compensate the fiber dispersion. As shown in the inset of Fig. 4, the QBERs at $75.8 \mathrm{~km}$ and $101.1 \mathrm{~km}$ have now been reduced to $6.30 \%$ and $7.80 \%$, respectively, well below the security limit, thus allowing secure key exchange to take place. The secure key rate is determined to be $19.0 \mathrm{kbps}$ and $2.88 \mathrm{kbps}$ for 75.8 and $101.1 \mathrm{~km}$ respectively, as shown in Fig. 4.

Finally, we would like to point out that the experiment described here is only a proof-of-principle demonstration. To achieve a complete GHz QKD system, there are still remaining challenges, such as remote synchronization, high speed real-time error correction and privacy amplification, to be addressed.

In summary, we have demonstrated $\mathrm{GHz}$ QKD with high bit rate using self-differencing InGaAs APDs over up to $101.1 \mathrm{~km}$ standard telecom fiber. The secure bit rate is determined to be $2.37 \mathrm{Mbps}$ for a $5.6 \mathrm{~km}$ long fiber, and decreases exponentially to $2.88 \mathrm{kbps}$ for $101.1 \mathrm{~km}$. We conclude that that InGaAs APDs are suitable for long distance $\mathrm{GHz}$ QKD systems. These results open the prospect of low cost and practical high bandwidth $\mathrm{GHz}$ QKD systems for future applications.
[1] C. H. Bennett and G. Brassard, Proceedings of the IEEE International Conference on Computers, Systems and Signal Processing, Bangalore, India, 1984, pp. 175-179.
[2] P. D. Townsend, Electron. Lett. 33, 188(1997).

[3] N. Gisin, G. Ribordy, W. Tittel, and H. Zbinden, Rev. Mod. Phys. 74, 145(2002). 
[4] M. Dušek, N. Lütkenhaus, M. Hendrych, in Progress in Optics, Vol. 49, pp. 381, edited by E. Wolf (Elsevier 2006).

[5] J. C. Bienfang, A. J. Gross, A. Mink, B. J. Hershman, A. Nakassis, X. Tang, R. Lum, D. H. Su, C. W. Clark, C. J. Williams, E. W. Hagley, and J. Wen, Opt. Express 12, 2011(2004).

[6] K. J. Gordon, V. Fernandez, G. S. Buller, I. Rech, S. D. Cova, and P. D. Townsend, Opt. Express 13, 3015(2005).

[7] H. Takesue, S. W. Nam, Q. Zhang, R. H. Hadfield, T. Honjo, K. Tamaki, and Y. Yamamoto, Nature Photonics 1, 343(2007).

[8] R. T. Thew, S. Tanzilli, L. Krainer, S. C. Zeller, A A. Rochas, I. Rech, S. Cova, H. Zbinden, and N. Gisin, New J. Phys. 8, 32(2006).

[9] E. Diamanti, H. Takesue, C. Langrock, M. M. Fejer, and Y. Yamamoto, Opt. Express 14, 13073(2006).

[10] H. Xu, L. Ma, A. Mink, B. Hershman, and X. Tang, Opt. Express 15, 7247(2007).

[11] N. Namekata, G. Fuji, S. Inoue, T. Honjo, and H. Takesue, Appl. Phys. Lett. 91, 011112(2007).

[12] C. Gobby, Z. L. Yuan and A. J. Shields, Appl. Phys. Lett.
84, 3762(2004).

[13] A. Yoshizawa, R. Kaji, and H. Tsuchida, Jpn. J. Appl. Phys., Part I 43, L735(2004).

[14] Z. L. Yuan, A. W. Sharpe, and A. J. Shields, Appl. Phys. Lett. 90, 011118(2007).

[15] G. Ribordy, J. D. Gautier, H. Zbinden, and N. Gisin, Appl. Opt. 37, 2272(1998).

[16] Z. L. Yuan, B. E. Kardynal, A. W. Sharpe, and A. J. Shields, Appl. Phys. Lett. 91, 041114(2007).

[17] P. W. Shor and J. Preskill, Phys. Rev. Lett. 85, 441(2000).

[18] C. Shannon, Bell Syst. Tech. J. 27, 379(1948).

[19] D. Gottesman, H. K. Lo, N. Lütkenhaus, and J. Preskill, Quant. Inf. Comput. 4, 325(2004).

[20] M. Dušek, O. Haderka, adn M. Hendrych, Opt. Commun. 169, 103(1999).

[21] G. Brassard, N. Lütkenhaus, T. Mor, and B. C. Sanders, Phys. Rev. Lett. 85, 1330(2000).

[22] H. K. Lo, X. Ma and K. Chen, Phys. Rev. Lett. 94, 230504(2005).

[23] X. B. Wang, Phys. Rev. Lett. 94, 230503(2005). 